\title{
Skin Hyperpigmentation Index Facilitating Quantification of Hyperpigmentation in Clinical Practice
}

\author{
Simon Bossart ${ }^{a} \quad$ Albert-Adrien Ramelet $^{\mathrm{a}} \quad$ Torsten Willenberg $^{\mathrm{b}}$ \\ Simone Cazzaniga ${ }^{\mathrm{a}, \mathrm{c}}$ Marc Baumgartner ${ }^{\mathrm{a}}$ Kristine Heidemeyer $^{\mathrm{a}}$ \\ Robert E. Hunger ${ }^{\mathrm{a}}$ S. Morteza Seyed Jafari ${ }^{\mathrm{a}}$ \\ ${ }^{a}$ Department of Dermatology, Inselspital, Bern University Hospital, University of Bern, Bern, Switzerland; \\ ${ }^{b}$ Gefässzentrum Bern, VASC, Lindehofspital Bern, Bern, Switzerland; ' Centro Studi GISED, Bergamo, Italy
}

\section{Keywords}

Skin hyperpigmentation · Skin hyperpigmentation index ·

Quantification

\section{Dear Editor}

Hyperpigmentation is a darkening of the skin, mostly caused by increased production of melanin due to melanocyte activation or hemosiderin deposits in the skin [1, 2]. As pigmentary disorders in dermatology can be disfiguring for patients and affect their quality of life, a brightening therapy is often demanded [3]. In order to measure the efficacy of various treatment modalities, skin hyperpigmentation should be evaluated quantitatively $[2,3]$. In addition, the hyperpigmentation of skin can also be a cutaneous manifestation of systemic diseases or conditions, which raises the need for a proper quantification of skin hyperpigmentation to monitor disease progression [1-3].

Although histopathological conclusions can be drawn about hyperpigmentation, there are only a few methods that allow clinical quantification of skin hyperpigmentation $[4,5]$. Many methods fail due to the lack of standardized conditions such as different illumination, different distances, and use of different devices.

As a result, we recently presented an advanced digital image processing system called the "skin hyperpigmenta-

karger@karger.com

(c) 2020 S. Karger AG, Basel

www.karger.com/drm

Karger! tion index" (SHI) for the automated quantification of skin hyperpigmentation. The SHI is a program with a deconvolution of color and an image histogram profiling of brown pixel intensities for the automated evaluation of quantitative skin pigmentation. It describes the ratio of two scores, namely the hyperpigmented skin of the affected area and normal sun-protected skin from the same patient (technical details were presented elsewhere) [2]. The SHI ranges from 1 (no hyperpigmentation) to 4 (maximum hyperpigmentation). We defined scores between 1 and 2 as light hyperpigmentation, 2-3 as medium hyperpigmentation, and scores between 3 and 4 as severe hyperpigmentation.

To ensure standardized presettings (same exposure and distance), the images should be taken with a dermoscope attachment for the camera (photo camera or smartphone). However, the use of standardized photos without a dermoscope attachment is possible, but results may be less accurate [2].

To facilitate this quantification, an online free SHI Calculator that is accessible to all practitioners is provided: https://shi.skinimageanalysis.com/.

For better illustration, we tested our method with two different cameras and dermoscope attachments on a 62 -year-old patient with pigmented purpuric dermatosis (capillaritis) of both legs (Fig. 1a). 
Fig. 1. a Overview image of a 62-year-old patient with a pigmented purpuric dermatosis (capillaritis) of both legs. Encircled area shows the location of the dermoscope image of the darkest hyperpigmented area. A reference image of sun-protected skin was taken on the lower back (not shown here). b Dermoscope image of hyperpigmentation of the right ankle. The photo was taken with an iPhone 7 camera with a dermoscope attachment of DermLite DL1 for iPhone. A reference image was taken from a sun-protected spot on the lower back (not shown here). The calculated SHI was 1.61. c Dermoscope image of hyperpigmentation of the right foot. The photo was taken with a Nikon D810 digital camera with a DermLite 3 dermoscopy lens. A reference image was taken from a sun-protected spot on the lower back (not shown here). The calculated SHI was 1.73.
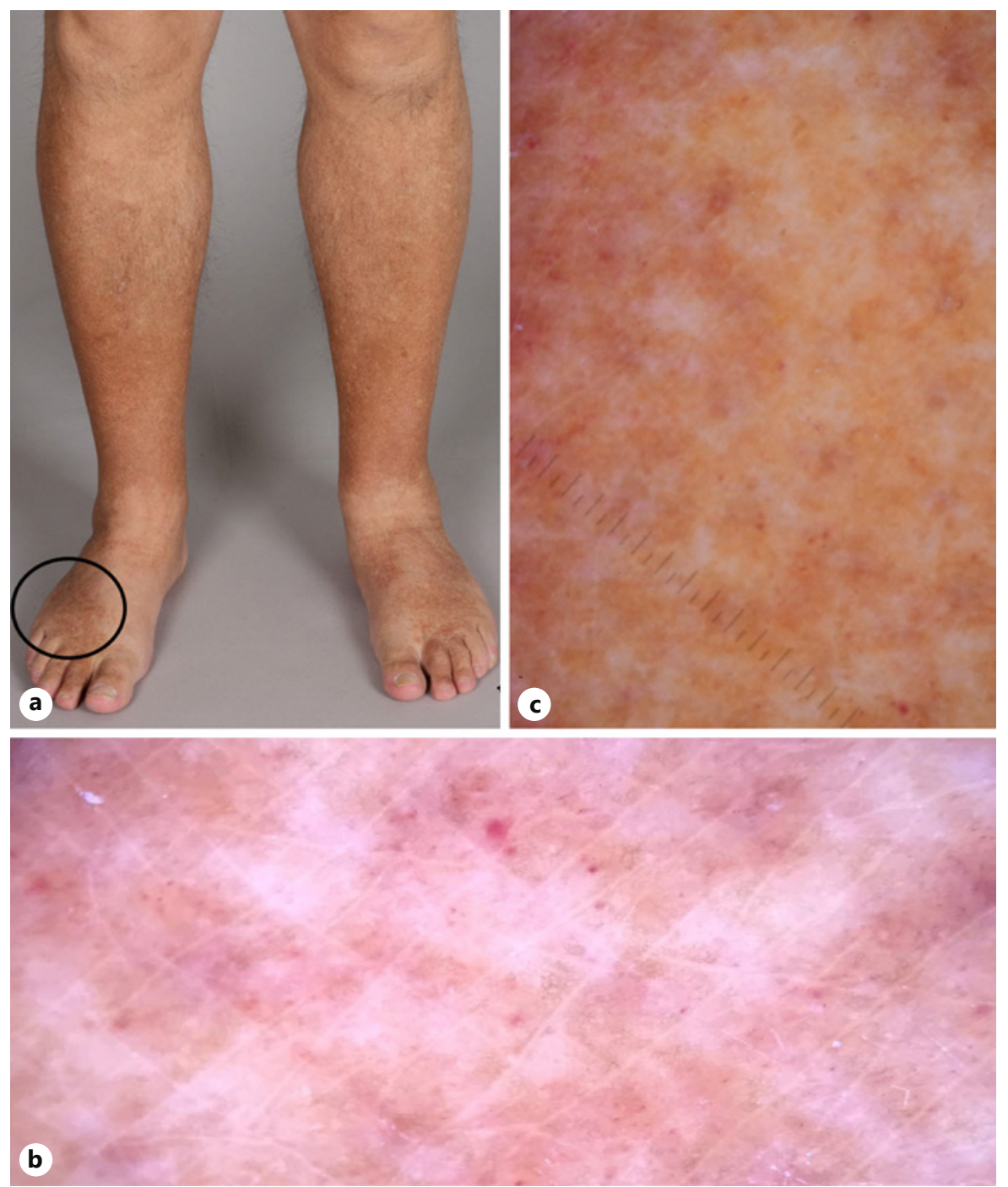

The image was recorded with a Smartphone iPhone 7 with a dermoscope attachment of DermLite DL1 for iPhone (Fig. 1b). The calculated SHI was 1.61. At the same location, photos were taken with a Nikon D810 digital camera with DermLite 3 dermoscopy lens (Fig. 1c). The calculated SHI was 1.73 . Both results showed a slight hyperpigmentation in the examined pictures. The SHI was calculated on https://shi.skinimageanalysis.com/.

With the SHI we have developed a new fully automated method for the quantitative assessment of skin pigmentation, which can be easily used for any skin type with any type of hyperpigmentation with different devices (dermoscope attachments and camera) [2].

This allows an easy, fast, and standardized quantification of skin hyperpigmentation, which can be very useful as follow-up control and planning of a whitening therapy.

\section{Key Message}

The skin hyperpigmentation index facilitates quantification of hyperpigmentation in clinical practice.

\section{Statement of Ethics}

The patient mentioned in this paper gave written informed consent for the publication of their case (including the publication of images).

\section{Conflict of Interest Statement}

The authors declare no conflicts of interest. 


\section{Funding Sources}

The authors declare no funding sources.

\section{Author Contributions}

All authors participated in analyzing the data. S.B. and S.M.S.J. wrote the paper. All authors read and approved the manuscript.

\section{References}

1 Chang MW. Disorders of hyperpigmentation. In: Bolognia JL, Schaffer JV, Cerroni L, editors. Dermatology. 4th ed. Amsterdam: Elsevier; 2018. p. 1115-43.

2 Bossart S, Cazzaniga S, Willenberg T, Ramelet AA, Baumgartner M, Hunger RE, et al. Skin hyperpigmentation index: a new practical method for unbiased automated quantification of skin hyperpigmentation. J Eur Acad Dermatol Venereol. 2020 Jul;34(7):e334e336.
3 Yadav A, Garg T, Mandal AK, Chander R. Quality of life in patients with acquired pigmentation: an observational study. J Cosmet Dermatol. 2018 Dec;17(6):1293-4.

4 Becker F, Fourgeau P, Carpentier PH, Ouchène A. Quantification of early cutaneous manifestations of chronic venous insufficiency by automated analysis of photographic images: feasibility and technical considerations. Phlebology. 2018 Jun;33(5):309-14.

5 Tiwary SK, Kumar PK, Dhameeja N, et al. Assessment and grading of pigmentation in chronic venous insufficiency. Phlebology. 2020 Jul;35(6):394-401 